\title{
Faire vivre une expérience d'évaluation mutuelle en formation initiale : quelle transposition vers les pratiques d'enseignement projetées ?
}

\author{
Christelle GOFFIN et Annick FAGNANT \\ Université de Liège, Belgique
}

\section{Résumé}

Dans une étude préalable menée en Belgique francophone (Fagnant et Goffin, à paraître), nous avons notamment montré que les futurs enseignants du secondaire supérieur (grades 10-12) témoignaient d'expériences limitées en matière d'évaluation formative : leur vécu en tant qu'élève faisait davantage état de pratiques d'auto-notation que de réelles expériences d'autoévaluation. Voulant prendre en compte le décalage entre le cadre légal qui prescrit la mise en œuvre d'évaluations formatives et la réalité des classes où ce type d'évaluation semble être peu pratiqué, nous avons tenté d'agir dès la formation initiale. Ainsi, nous avons fait vivre à ces futurs enseignants une expérience d'évaluation mutuelle en tant qu'étudiant universitaire dans un cours pluridisciplinaire tout en les faisant réfléchir à la transposition de ces acquis vers leurs pratiques de stage en tant qu'enseignant d'une discipline donnée. Cet article présente le dispositif mis en place, tout en y portant un regard critique documenté par deux sources d'informations. La première concerne les commentaires rédigés par les étudiants sur ce qu'ils ont retenu du dispositif expérimenté. La seconde porte sur les pratiques d'évaluation que les futurs enseignants ont développées ou pourraient développer en classe avec leurs élèves, en lien avec l'expérience vécue en formation. Les analyses mettent en évidence que cette expérience a pu, dans une certaine mesure, nourrir leurs processus d'apprentissage et leur développement professionnel.

\section{Mots-clés}

Évaluation mutuelle, formation d'enseignants, pratiques d'évaluation, apprentissage de l'évaluation, isomorphisme.

\begin{abstract}
In a preliminary study conducted in the French-speaking community of Belgium (Fagnant \& Goffin, forthcoming), we showed that future upper secondary school teachers (grades 10-12) reported limited experiences in formative evaluation during their schooling: they mentioned more self-grading practices than actual experiences of self-assessment. In order to deal with the discrepancy between the institutional context that prescribes the implementation of formative evaluation and the reality of the classes, where this type of practice is still uncommon, we chose to act at the level of initial training. We thus proposed to the future teachers an experience of peer-to-peer assessment as academic students in a multidisciplinary course, while making them reflect on possible transpositions of this knowledge into their practice as teachers in a given discipline. This article presents the implemented device and its critical assessment, documented by two sources of information. The first concerns the comments written by the students on this experience. The second relates to the assessment practices they developed or could develop as teachers, in relation to this experience. To some extent, the study shows positive results on their learning processes and their professional development.
\end{abstract}

\section{Keywords}

Peer to peer assessment, teacher training, assessment practices, learning of assessment, isomorphism. 


\section{Introduction}

Comme dans de nombreux pays (Ocdé, 2005), la pratique de l'évaluation formative est vivement recommandée en Belgique francophone depuis de nombreuses années, et ceci tant dans les textes officiels décrivant les attentes du système scolaire (Décret « Missions ", 1997) qu'au niveau de la formation initiale d'enseignants (Paquay, 1994). Des études menées notamment en France, en Suisse romande et en Belgique francophone (Issaieva et Crahay, 2010 ; Issaieva, Yerly, Petkova, Marbaise, et Crahay, 2015 ; Luisoni et Monnard, 2015 ; Soussi, Ducrey, Ferrez, Nidegger et Viry, 2006) ont montré que les enseignants semblaient reconnaître l'intérêt des évaluations formatives pour soutenir les apprentissages des élèves, mais qu'ils étaient toutefois relativement peu enclins à mettre en œuvre ce type d'approche dans leurs classes (Soussi et al., 2010 ; Younès et Gaime, 2012). D'après l'enquête Thalis 2013 (Ocdé, 2014), si l'autoévaluation est très fréquente dans certains pays comme l'Angleterre ou le Canada, elle est très peu pratiquée dans d'autres, comme en France notamment où seuls $16,5 \%$ des enseignants du premier cycle du secondaire déclarent utiliser cette modalité impliquant l'élève dans le processus d'évaluation (Cnesco, 2014). La Belgique francophone n'a pas participé à cette enquête, mais, nous basant sur une étude que nous avons menée en 2015-2016 auprès de futurs enseignants du secondaire supérieur (grades 10-12) (Fagnant et Goffin, à paraître), nous pouvons penser que peu d'autoévaluations sont mises en place dans les classes belges francophones.

Dans cette étude, nous avions, entre autres, demandé à ces futurs enseignants de se positionner face à des affirmations visant à évaluer l'intérêt qu'ils portaient aux évaluations formatives, mais aussi de décrire des expériences auxquelles ils avaient été confrontés durant leur parcours scolaire. S'ils reconnaissaient volontiers l'intérêt de pratiquer des évaluations formatives, leur vécu en tant qu'élève concernant ces évaluations (et plus particulièrement ici, l'autoévaluation et l'évaluation mutuelle) s'est révélé particulièrement limité. En effet, ils faisaient principalement état de pratiques d'auto-notation (Rey et Feyfant, 2014), relatant davantage des procédures de correction (par exemple, noter leur propre copie en s'appuyant sur la grille de critères fournie par l'enseignant) que de réelles pratiques d'autoévaluation potentiellement porteuses d'apprentissage et invitant à un véritable recul métacognitif (Campanale, 2007).

Partant de l'hypothèse selon laquelle le vécu en tant qu'élève (socialisation secondaire Campanale, 2007 ; Crahay, Wanlin, Issaieva et Laduron, 2010 ; Vause, 2010) influence les conceptions des futurs enseignants, on peut craindre que les accords de principes formulés face aux affirmations qui leur étaient proposées traduisent une forme de «désirabilité sociale » (Butori et Parguel, 2010), mais que leurs conceptions profondes soient en réalité moins enthousiastes à l'égard de l'évaluation formative. Suite à leur vécu en tant qu'élève, on peut en effet s'attendre à ce que les futurs enseignants aient en réalité une vision de l'évaluation formative assez « classique » ou peu «élargie » (Allal et Mottier Lopez, 2005 ; Morissette, 2010) et qu'ils imaginent finalement peu comment intégrer ces pratiques dans les classes.

Face à ces divers constats et nous appuyant sur un principe d'isomorphisme ou d'homologie (Campanale, 2007; Vanhule, 2002), nous avons décidé de faire vivre une expérience d'évaluation mutuelle (Bourgeois et Laveault, 2015) à ces futurs enseignants, tout en les faisant réfléchir à la transposition de ces acquis vers leurs pratiques. Il s'agissait ainsi d'envisager "l'évaluation comme objet d'apprentissage et comme outil de développement professionnel » (Fagnant, Etienne, Mottier Lopez et Hindryckx, soumis). 
L'objet du présent article est de faire part de cette expérience et de quelques résultats tirés de l'évaluation de celle-ci en termes de transpositions entre l'expérience vécue et les pratiques d'enseignement projetées. En accord avec ce numéro thématique, une précision des contextes dans lesquels l'étude s'inscrit s'impose en préambule.

\section{Les différents contextes en interaction}

De façon certes simplifiée, nous allons situer la présente étude dans quatre contextes en étroite interaction : le système scolaire en Belgique francophone (contexte 1), la formation des enseignants, constituée de cours (contexte 2) et de stages (contexte 3 ) et, enfin, les écoles et les classes où exerceront les enseignants lorsqu'ils seront diplômés (contexte 4).

Le système scolaire (contexte 1) est notamment régi par le décret "Missions » (1997) " définissant les missions prioritaires de l'enseignement fondamental et de l'enseignement secondaire et organisant les structures propres à les atteindre ». Celui-ci prône la pratique de l'évaluation formative (article 15) définie comme une "évaluation effectuée en cours d'activité et visant à apprécier le progrès accompli par l'élève et à comprendre la nature des difficultés qu'il rencontre lors d'un apprentissage ; elle a pour but d'améliorer, de corriger ou de réajuster le cheminement de l'élève ; elle se fonde en partie sur l'auto-évaluation » (p.4). Certaines tensions sont sans doute perceptibles entre cette vision d'une évaluation au service des apprentissages et le contexte historique d'un système scolaire fortement marqué par le redoublement et les relents d'une logique méritocratique conduisant au classement et à la sélection des élèves (Crahay, 2013). On imagine aisément que ces tensions peuvent constituer un frein à la mise en œuvre de réelles pratiques d'évaluation formative (Issaieva et al., 2015).

En Belgique francophone, le titre pédagogique requis pour enseigner dans le secondaire supérieur (grades 10-12, élèves âgés de 15 à 18 ans environ) est l'agrégation de l'enseignement secondaire supérieur ou le master à finalité didactique. Cette formation universitaire est constituée de 15 ECTS de cours généraux et de 15 ECTS de stages et de cours spécifiques à la didactique de la discipline qui sera enseignée. Les cours généraux sont fréquentés par plus de 200 étudiants issus de 18 disciplines différentes (mathématiques, histoire, langues modernes, éducation physique, etc.). L'expérience d'évaluation mutuelle dont il est question ici a été mise en place en avril 2016. Elle s'est déroulée dans le contexte d'un cours général, et plus particulièrement lors d'une des séances de pratiques réflexives. Ces séances sont menées en petits groupes composés chacun d'environ 25 étudiants. Elles ont entre autres pour objectif de créer des connexions entre les cours théoriques, notamment pluridisciplinaires (contexte 2), et les stages (contexte 3 ) où les étudiants doivent ouvrer en tant qu'enseignant d'une discipline donnée.

Enfin, dans le cadre de notre étude, nous discuterons des transferts envisagés vers les pratiques professionnelles futures que les étudiants, une fois diplômés, seraient susceptibles de mettre en œuvre dans les classes dans lesquelles ils exerceront en tant qu'enseignant (contexte 4).

\section{Le dispositif d'évaluation mutuelle mis en place}

Si l'évaluation mutuelle consiste à ce que deux ou plusieurs apprenants s'impliquent activement dans l'évaluation de leurs productions respectives, l'objectif est in fine de développer l'autoévaluation (Mottier Lopez, 2015). L'autoévaluation, qui s'inscrit comme 
une modalité d'évaluation formative au service de l'apprentissage (Morissette, 2010), implique «d'encourager les élèves à réfléchir sur ce qu'ils ont appris, à chercher les moyens d'améliorer leur apprentissage, et à planifier ce qui leur permet de progresser en tant qu'apprenant et d'atteindre leurs objectifs » (Rey et Feyfant, 2014 : 28).

Par ailleurs, c'est notamment en référence au principe d'homologie ou d'isomorphisme que nous avons voulu que les futurs enseignants vivent réellement une évaluation mutuelle. Appliqué à l'éducation, ce principe postule que, en faisant vivre aux futurs enseignants une activité proche de celle qu'ils auront à faire vivre aux élèves, ils transféreront plus facilement cette activité dans leurs pratiques. Un tel dispositif s'appuie ainsi sur l'hypothèse selon laquelle les modalités de formation seraient plus puissantes que les contenus eux-mêmes (Campanale, 2007). Soulignons encore qu'il ne suffit pas non plus de vivre une activité pour que la transposition dans les pratiques de classe soit automatique. Il est en effet essentiel de consacrer du temps pour analyser l'expérience vécue (Vanhulle, 2002): les enjeux de l'activité, ce qui a été fait et comment cela a été mis en place, les difficultés éventuellement rencontrées, et ce que cela implique si des activités similaires étaient construites pour leurs futurs élèves, avec la possibilité d'imager des variantes...

\subsection{Description du dispositif}

Avant la séance consacrée à l'évaluation mutuelle, nous avons demandé aux étudiants d'analyser, à l'aide de l'outil CompAS (Parmentier et Paquay, 2002) ${ }^{1}$, une séquence de cours qu'ils avaient mise en œuvre en stage. Ils devaient rédiger un texte présentant cette analyse de façon à disposer d'un matériau qui servirait de base à un dialogue évaluatif constructif. Nous les avons informés que ce travail préparatoire serait lu et évalué par un autre étudiant lors de ladite séance et qu'il pourrait ensuite être retravaillé en vue de figurer dans le travail réflexif final, qui fait partie de l'évaluation certificative du cours.

Concrètement, une partie de ce travail réflexif final porte sur l'analyse d'une séquence d'enseignement/apprentissage que les étudiants ont mise en œuvre en stage. Ils doivent rédiger une présentation de la séquence choisie en la contextualisant et en décrivant les objectifs, le timing et les grandes étapes méthodologiques de celle-ci. Pour analyser cette séquence, ils sont amenés à compléter l'outil CompAS et à choisir deux facettes dites satisfaisantes (c'est-à-dire rencontrées dans leur séquence) et deux facettes dites interpellantes (c'est-à-dire peu rencontrées alors qu'elles leur semblent importantes). Dans le premier cas, ils sont invités à expliquer ce qu'ils ont mis concrètement en œuvre en classe pour rencontrer ces facettes; dans le second cas, ils sont tenus d'expliquer ce qu'ils pourraient mettre concrètement en œuvre en classe afin de les rencontrer davantage. Parmi les quatre facettes, les étudiants sont contraints d'exploiter la facette relative à l'évaluation. Quant au travail préparatoire, il devait être réalisé pour deux des quatre facettes: une satisfaisante et une interpellante.

\footnotetext{
${ }^{1}$ L'outil CompAS a été conçu par des chercheurs de l'Université catholique de Louvain, en collaboration avec des formateurs d'enseignants et des enseignants du secondaire. Cet outil a été construit en vue d'apprécier comment les dispositifs d'enseignement favorisent le développement de compétences par les apprenants. Les auteurs ont élaboré un modèle pour la construction de compétences qui intègre et articule les différentes facettes des activités d'enseignement/apprentissage. Parmi ces facettes, l'une concerne l'évaluation et s'intéresse notamment à l'implication des élèves dans le processus.
} 
Lors de la séance d'évaluation mutuelle, nous leur avons demandé, comme annoncé, de lire le texte rédigé par un de leurs condisciples et d'évaluer ce travail préparatoire sur la base d'une grille d'évaluation constituée de critères et d'indicateurs. Cette grille avait préalablement été construite et validée par les six formatrices impliquées dans le cours et serait ensuite utilisée pour l'évaluation certificative de cette partie du travail réflexif final.

Avant de réaliser cette évaluation mutuelle en duo pluridisciplinaire, nous avons présenté la grille d'évaluation aux étudiants. Comme le recommandent Bourgeois et Laveault (2015), nous avons veillé à la compréhension des objectifs attendus, des critères et des indicateurs. Pour évaluer le travail de leur condisciple, les étudiants devaient non seulement identifier les forces et les faiblesses en attribuant une appréciation $(-/ \pm /+)$ face à chaque indicateur de la grille, mais ils devaient également préciser sur quels éléments concrets ils fondaient leurs appréciations. Ils devaient aussi formuler des pistes pour aider l'étudiant concerné à améliorer son travail. Nous avons insisté sur la nécessité de justifier les appréciations données et d'indiquer des pistes à mettre en œuvre pour rencontrer davantage les critères et les indicateurs, afin que les étudiants ne se contentent pas d'attribuer une simple appréciation. En effet, si tel était le cas, l'évaluation mutuelle tomberait sans doute dans les mêmes travers que 1'auto-notation: elle risquerait de ne pas conduire à un véritable recul métacognitif et à de réelles régulations. A contrario, les justifications des forces et faiblesses et les pistes d'amélioration devraient susciter les interactions verbales favorisant les régulations (Campanale, 2007). Après avoir pris connaissance de l'évaluation réalisée par le pair, les duos ont eu l'occasion d'en discuter et de retravailler leur travail préparatoire.

Par la suite, avec l'ensemble du petit groupe pluridisciplinaire, nous sommes alors revenues sur la méthodologie vécue afin de faire réfléchir les étudiants sur les éléments auxquels ils devaient être attentifs pour transférer cette expérience vers leurs futures pratiques d'enseignement (Bourgeois et Laveault, 2015 ; Vanhulle, 2002). Pour terminer la séance, nous avons amené les étudiants à poursuivre le travail initialement demandé en réfléchissant à la façon dont ils pourraient intégrer une évaluation dans la séquence de cours analysée ou, s'ils en avaient déjà prévue une, d'expliciter ce qu'ils avaient mis en œuvre.

\subsection{Objectifs visés par le dispositif et liens entre les contextes}

Le dispositif visait un double objectif, assorti de trois boucles possibles de rétroaction dans différents contextes.

Premièrement, il s'agissait d'amener les futurs enseignants à participer, en tant qu'étudiants universitaires, à une évaluation formative en vue de préparer l'évaluation certificative. Dans ce cas-ci, on peut situer l'objectif comme une boucle de rétroaction qui agit au sein même du contexte 2 (le cours lui-même).

Deuxièmement, en référence au principe d'homologie ou d'isomorphisme (Campanale, 2007 ; Vanhule, 2002), participer à cette expérience d'évaluation mutuelle (mais aussi prendre du recul et analyser ce dispositif) visait à initier de nouvelles pratiques. Deux boucles de rétroaction sont ici envisagées: d'une part, une possible intégration d'expériences d'évaluation formative au sein même de la séquence de cours qu'ils ont analysée (liens entre le contexte 2 - cours général pluridisciplinaire - et le contexte 3 - stage d'enseignement disciplinaire -, explicitement demandés dans les consignes du travail réflexif final) et, d'autre part, une réflexion quant à de possibles transpositions vers leurs pratiques futures, lorsqu'ils auront terminé leur formation et qu'ils prendront leur fonction en tant qu'enseignant dans une 
école (liens escomptés entre la formation dans son ensemble - contextes 2 et 3 - et les futures pratiques professionnelles - contexte 4 ).

\section{L'évaluation du dispositif mis en place}

Cette évaluation prend la forme d'un regard critique documenté par deux sources d'informations. La première, qui est centrale, concerne les commentaires rédigés par les étudiants suite à l'expérience d'évaluation mutuelle vécue. La seconde, intervenant à titre illustratif, porte sur les pratiques d'évaluation que les futurs enseignants ont développées ou pourraient développer en classe, pratiques décrites dans les travaux réflexifs de fin d'année.

\subsection{Que nous racontent les étudiants au sujet de cette expérience?}

Nous avons demandé aux étudiants d'expliquer en quelques lignes ce qu'ils avaient retenu de l'expérience d'évaluation mutuelle vécue. Cette consigne était volontairement vague et non orientée de façon à permettre aux étudiants de choisir les enjeux qui leur semblaient importants ou non de souligner.

En nous inspirant de l'approche inductive proposée par Blais et Martineau (2006) ${ }^{2}$, nous avons analysé les 181 commentaires recueillis en vue de faire ressortir les enjeux mentionnés spontanément par les étudiants. Notons que, si certains étudiants n'ont indiqué qu'un enjeu, d'autres ont fait part de plusieurs. Nous avons réparti les catégories ainsi créées selon le contexte auquel elles faisaient référence : le fait de vivre une évaluation mutuelle, soit en tant qu'étudiant universitaire devant préparer l'évaluation certificative d'un cours donné (rétroaction au sein du contexte 2), soit en tant que futur enseignant cherchant à transposer l'expérience vécue vers ses pratiques (transposition du contexte 2 - cours général pluridisciplinaire - vers le contexte 3 - stage d'enseignement disciplinaire - ou 4 - pratiques professionnelles futures).

\subsubsection{Participer à une évaluation formative en tant qu'étudiant universitaire (boucle de rétroaction au sein du contexte 2)}

Les trois premières catégories se centrent sur les qualités du «produit attendu» pour l'évaluation certificative. Les commentaires font référence au caractère formatif de l'expérience vécue, soit en se cantonnant au versant « informatif» (des consignes du travail à réaliser ou des qualités de la première ébauche de ce travail), soit en mentionnant explicitement les versants « information » et « régulation » associés à ce type de pratique :

- Une série de déclarations portent sur l'idée selon laquelle l'évaluation mutuelle a engendré une meilleure compréhension du travail attendu : les étudiants sont au nombre de 32 à déclarer avoir été mieux informés des attentes (par exemple, J'ai apprécié le fait de me retrouver à mon tour "dans la peau de l'évaluateur », car cela m'a aidé à mieux cerner et comprendre ce qu'il était attendu de ce travail - Étudiant en langues modernes).

- Pour 30 étudiants, l'évaluation mutuelle a permis d'être informés des points forts et/ou à améliorer de leur travail préparatoire. Ces étudiants ne disent toutefois pas explicitement ce qu'ils font ou feront de ce feedback formatif (par

\footnotetext{
${ }^{2}$ Plus concrètement, nous avons lu chaque commentaire plusieurs fois jusqu'à avoir une vue d'ensemble des enjeux mentionnés. Nous avons ensuite identifié les segments de commentaires qui constituaient des unités de sens et, sur cette base, nous avons créé des catégories. Chaque segment de commentaires a alors été intégré dans une catégorie déjà créée ou, le cas échéant, a conduit à créer une nouvelle catégorie.
} 
exemple, Le regard de l'autre est toujours positif car il permet de mettre l'accent sur les faiblesses et les points forts de notre production - Étudiant en histoire de l'art).

- Cent étudiants rapportent avoir été informés et avoir pu réguler leur travail au niveau du "contenu ». Il est à noter que, si cette régulation se réalise, pour la grande majorité de ces étudiants, grâce aux commentaires reçus (par exemple, Je me suis référée aux commentaires de mon partenaire afin de corriger les deux facettes déjà écrites et de poursuivre le travail grâce à ses conseils - Étudiant en langues romanes), certains d'entre eux mentionnent qu'ils se sont régulés grâce à la lecture de l'autre travail (par exemple, Cela nous a aussi permis de voir la production d'autres étudiants et donc de voir des pistes de réalisation pour les parties qui nous semblaient plus obscures Étudiant en chimie).

Les deux dernières catégories se réfèrent moins directement aux qualités du "produit attendu », mais elles n'en demeurent pas moins liées à leur position d'étudiant ayant vécu une expérience singulière dans un cours (la rétroaction reste au sein du contexte 2) :

- Six étudiants rapportent avoir été informés au niveau des méthodes de travail cette fois (par exemple, Grâce à cette expérience, j'ai également pu voir comment les autres avaient procédé pour réaliser leur travail et confronter leur méthode à la mienne - Étudiant en langues classiques). L'un d'entre eux évoque explicitement avoir pu réguler sa façon de travailler.

- Une douzaine d'étudiants déclarent que l'évaluation mutuelle les a rassurés (par exemple, Mon collègue m'a conforté dans certains choix que j'avais faits - Étudiant en histoire de l'art; Il m'a donné confiance dans ma démarche Étudiant en histoire de l'art).

4.1.2. Vivre une expérience d'évaluation formative en tant que futur enseignant (transposition du contexte 2 vers les contextes 3 et 4 )

Les deux premières catégories évoquent explicitement l'objet d'apprentissage visé au travers de l'expérience vécue : l'évaluation formative dans une vision élargie et, singulièrement ici, l'évaluation mutuelle comme une pratique permettant d'impliquer l'élève dans le processus d'évaluation :

- Trois étudiants déclarent spontanément que l'expérience vécue leur a permis d'élargir leurs conceptions en matière d'évaluation : ils ont expliqué avoir mieux compris ce qu'était une évaluation mutuelle et/ou concevoir l'évaluation formative dans sa perspective élargie (par exemple, L'évaluation mutuelle m'a permis de comprendre en quoi consistait une évaluation mutuelle - Étudiant en biologie; Cela m'a permis de me rappeler que l'évaluation formative peut revêtir des formes différentes que celle de l'évaluation classique - Étudiant en chimie).

- Les propos de 40 étudiants montrent que l'expérience vécue les a conduits à réfléchir à une possible transposition de dispositifs d'évaluation mutuelle dans leurs futures classes. Si la moitié d'entre eux a plutôt précisé l'intérêt pour les élèves (par exemple, La méthode est intéressante : réaliser cet exercice en classe avec les élèves pourrait être pertinent. En effet, en réalisant cet exercice, l'élève apprend à évaluer, à vérifier que les consignes sont respectées. Cet apprentissage pourra lui être utile pour s'autoévaluer Étudiant en sciences sociales; Je pense que c'est une bonne idée à appliquer 
en classe, cela pousse l'élève à s'investir puisqu'il doit aider son camarade en l'évaluant au mieux et en plus, la discussion qui vient après peut apporter des éclaircissements sur certains points de la matière moins bien/pas compris Étudiant en chimie), l'autre moitié s'est davantage placée du point de vue de l'enseignant (par exemple, Avoir vécu cette évaluation mutuelle m'a paru très utile car je saurai à l'avenir comment l'organiser au sein de mes futures classes - Étudiant en langues modernes; Jusqu'à ce que je ne prenne moimême part à cette expérience, je ne me rendais pas vraiment compte de la manière dont je pouvais m'y prendre pour appliquer ces méthodes dans mes futures classes - Étudiant en sciences économiques).

Si les deux dernières catégories laissent elles aussi entrevoir une possible régulation des pratiques d'enseignement, ce n'est pas au niveau de l'évaluation que la transposition se situe ici. La première catégorie reprend des propos qui restent spécifiques à la séquence de cours analysée et envisagent des modifications de celle-ci ("si c'était à refaire... »); la deuxième imagine de transposer des éléments découverts dans une discipline donnée dans des cours portant sur une autre discipline. Même s'ils ne relèvent pas du type de transposition explicitement visée dans les objectifs du dispositif, ils sont intéressants dans la mesure où ils amorcent également des liens entre le contexte 2 (cours pluridisciplinaire) et les contextes 3 (stages) et 4 (futures pratiques professionnelles) :

- Grâce aux commentaires reçus, l'évaluation mutuelle a permis à 4 étudiants d'être informés et d'envisager de réguler la séquence de cours elle-même (par exemple, Ces quelques faiblesses pourront dès lors faire l'objet d'un remaniement dans le cadre de la réutilisation de cette séquence - Étudiant en histoire).

- Grâce à la lecture de l'autre travail, l'évaluation mutuelle a permis à 15 étudiants de découvrir de nouvelles méthodes d'enseignement. Quatre d'entre eux envisagent explicitement de réguler leurs pratiques (par exemple, Par la lecture de la séquence de mon condisciple, j'ai pu avoir un aperçu de ses propres méthodologies. Celles-ci s'appliquaient à la physique, un cours scientifique. J'ai pu en retirer des éléments pour ma pratique dans le cadre du cours de biologie (situation-problème, expérimentation et modélisation Étudiant en bio).

\subsection{Que nous révèlent les travaux réflexifs ?}

Quelques étudiants décrivent une expérience d'évaluation formative qu'ils ont mise en œuvre en stage dans le cadre de la séquence de cours analysée. C'est le cas de l'étudiante suivante qui avait développé une évaluation mutuelle au départ d'une activité de production écrite :

- (...) J'ai ensuite redistribué les textes au hasard parmi la classe. Chacun était alors chargé de faire un commentaire sous forme de conseils sur la rédaction qu'il venait de recevoir en se basant sur des critères que j'avais inscrits au tableau (les péripéties sont-elles réalistes? La rédaction est-elle assez détaillée? etc.). Les élèves en tant qu'évaluateurs ont ainsi pu apporter un regard neuf et objectif sur la rédaction qu'il leur était demandé de commenter et ont pu dans certains cas émettre des idées supplémentaires. Les apprenants récupéraient ensuite leur rédaction ainsi que les conseils de leur condisciple et étaient amenés à modifier leur production initiale afin de me rendre une version définitive. (...) Grâce à cette tâche, les élèves ont été impliqués dans l'évaluation de leur camarade car ce sont leurs commentaires qui permettaient 
au condisciple d'améliorer son texte. D'autre part, cet exercice pose les élèves en évaluateur, dès lors ils visualisent mieux la manière dont se fait une correction et peuvent ainsi lors de prochains exercices porter un regard plus critique sur leur production (Étudiant en romanes).

La plupart des étudiants n'avaient toutefois pas mis en place d'évaluation formative durant leur stage; ce sont donc essentiellement des pistes explicitant ce qu'ils pourraient mettre concrètement en œuvre dans la séquence de cours analysée ("si c'était à refaire... »), qui sont décrites dans les travaux réflexifs. S'il faut reconnaître que beaucoup d'exemples sont peu développés, certaines initiatives intéressantes sont à souligner, comme l'illustre l'exemple repris ci-dessous :

- Une idée serait de mettre à disposition des élèves des outils tels qu'une grille de critères qu'ils devraient rencontrer lorsqu'ils présentent une réalisation d'exercices. (...) Deux grilles seraient disponibles : une pour les exercices systématiques et une pour l'analyse de problèmes plus complexes. (...) Il serait judicieux d'utiliser ce type de grilles pour chaque séquence de façon à habituer les élèves à travailler avec. Il est évident que, la première fois, le professeur devrait prévoir un temps nécessaire pour expliquer comment fonctionnent les grilles, à quoi elles servent, quelles informations elles vont apporter aux élèves,.... (...) Un élève serait alors envoyé au tableau (...). Le professeur prendrait ensuite la première grille (...) et analyserait la réponse proposée avec les élèves pour que ceux-ci puissent voir réellement comment elle fonctionne. Enfin pour certains exercices ciblés, il proposerait aux élèves de remplir la grille par eux-mêmes après la correction au tableau. Les élèves pourraient ainsi plus facilement voir les points qu'ils leur restent à améliorer (...) (Étudiant en chimie).

\section{Discussion et conclusion}

Partant du constat d'un décalage entre le cadre légal qui prescrit la mise en œuvre d'évaluations formatives et la réalité des classes où ce type d'évaluation semble être peu pratiqué (Fagnant et Goffin, à paraître), nous avons fait vivre aux futurs enseignants une expérience d'évaluation mutuelle en tant qu'étudiants universitaires dans un cours pluridisciplinaire. Un des objectifs du dispositif mis en place était de les faire réfléchir à la transposition de ces acquis vers leurs pratiques de stage et leurs pratiques professionnelles futures en tant qu'enseignants d'une discipline donnée.

Sur la base des commentaires rédigés spontanément par les étudiants suite à l'expérience d'évaluation mutuelle vécue, l'objectif de "préparer à l'évaluation certificative " est davantage rencontré que celui de «transposer de nouvelles pratiques d'évaluation ». En effet, 162 segments de commentaires portent explicitement sur les qualités du travail réflexif final, contre 40 qui font référence à un possible transfert. Malgré ce décalage entre les deux objectifs, ce résultat est en soi assez encourageant puisqu'un cinquième des étudiants semble donc envisager la possibilité de transposer l'expérience avec leurs futurs élèves.

Rappelons que les réflexions des futurs enseignants quant à la façon dont ils pourraient intégrer une évaluation formative dans la séquence de cours analysée sont dans la plupart des cas peu développées, donnant l'impression que les étudiants ont éprouvé des difficultés à concrétiser de telles pratiques d'évaluation. Par ailleurs, même si certains travaux font état d'exemples intéressants de possibles mises en œuvre d'évaluations formatives, nous ne 
devons pas oublier qu'ils ont été proposés dans le cadre d'une évaluation certificative et qu'ils pourraient simplement témoigner d'une volonté de répondre aux attentes des formateurs. Autrement dit, il est possible que ces exemples traduisent davantage une bonne compréhension des concepts impliqués et des objectifs visés par le dispositif mis en œuvre (ce qui n'est déjà pas négligeable), qu'ils ne constituent de réels indicateurs des pratiques professionnelles futures en matière d'évaluation.

Pour tenter d'expliquer le décalage entre les deux objectifs visés par le dispositif, ainsi que la difficulté liée à la concrétisation des exemples, il convient d'interroger la problématique de l'isomorphisme entre la situation vécue et les pratiques de classe en fonction des disciplines mobilisées. Naïvement, il serait louable de penser que le transfert sera plus facile dans une discipline "littéraire » que dans une discipline "scientifique » dans la mesure où la tâche réalisée s'appuie sur l'évaluation d'une production écrite. Toutefois, les commentaires invitant à une transposition de l'expérience vécue vers les pratiques de classe, ainsi que les exemples développés pour imaginer la mise en œuvre d'évaluations formatives en classe, sont rédigés par des étudiants issus de disciplines diversifiées. Même si ce transfert d'un cours général vers une discipline donnée est un élément positif, ne perdons pas de vue l'importance des spécificités et des enjeux disciplinaires pour la mise en œuvre de pratiques d'évaluation efficaces (Detroz, Crahay et Fagnant, 2017 ; Vantourout et Goasdoué, 2014). Ces aspects disciplinaires n'ont pas du tout été traités dans le contexte 2 (cours général pluridisciplinaire) alors qu'ils sont pourtant au cœur des pratiques dans les contextes 3 et 4 (pratiques d'enseignement en stage et, plus tard, dans la pratique professionnelle), ce qui est une limite importante de l'approche proposée (et une difficulté majeure d'un cours regroupant des étudiants issus de 18 disciplines différentes).

$\mathrm{Au}$ sein de la formation initiale toujours, l'écart entre les thématiques travaillées dans les cours généraux (contexte 2) et les préoccupations des étudiants lors de leurs stages d'enseignement (contexte 3) peut constituer un autre frein au développement des transpositions escomptées. Concrètement ici, alors que la réflexion sur la mise en œuvre d'évaluations formatives se focalise sur l'apprentissage et le développement des élèves (niveaux 4 et 5 des préoccupations de l'enseignant selon Durand, 1996), les étudiants se préoccupent essentiellement du maintien de l'ordre et de l'engagement des élèves dans la tâche (niveaux 1 et 2 selon Durand, 1996). Si on ajoute à cela l'idée selon laquelle les enseignants qui reçoivent les étudiants en stage dans leurs classes recourent peu aux évaluations formatives, la transposition de ce type de pratiques risque de se heurter à un nouvel obstacle. Dans le même ordre d'idées, même si les futurs enseignants adhéraient à une vision élargie de l'évaluation formative et même si la formation initiale (contextes 2 et 3 ) avait pu les convaincre des bienfaits et de la faisabilité de telles pratiques, une autre source de tensions pourrait apparaître lors de leur entrée dans le métier (contexte 4) puisqu'ils auront à collaborer avec des collègues qui ne partagent pas nécessairement la même vision qu'eux. Enfin, ils devront aussi chercher à conjuguer harmonieusement les visées formatives et certificatives des évaluations (Mottier Lopez, 2015), ce qui pourrait s'avérer encore plus complexe dans un système scolaire fortement marqué par l'évaluation-sanction et le redoublement (contexte 1).

Entre les préoccupations de survie (Huberman, 1989) et les possibles tensions ressenties lors de leur entrée dans le métier, certains enseignants débutants auraient tendance à revenir à des pratiques plus traditionnelles (De Stercke et al., 2010) voire à laisser resurgir d'anciennes conceptions qui semblaient pourtant avoir évolué durant la formation (Luisoni et Monnard, 2015). À l'instar de Devos et Paquay (2013), nous souhaitons nuancer ce propos qui donne 
l'impression que les enseignants débutants délaisseraient tout ce qu'ils ont appris en formation initiale au profit de ce qu'ils ont eux-mêmes connu en tant qu'élèves ou de pratiques en cours dans leur école. En effet, s'arrêter à un tel constat découragerait toute entreprise innovante en formation initiale. De façon plus optimiste, une fois cette période de survie dépassée, les enseignants devraient pouvoir grimper sur l'échelle des niveaux de préoccupation de Durand (1996) pour mettre en œuvre des pratiques se centrant sur l'apprentissage réel et le développement des élèves. S'ils ont vécu positivement certaines pratiques innovantes, ils devraient alors y repenser et chercher à les adapter au mieux à leur contexte de travail. À ce stade, à la fois motivés et conscients des difficultés inhérentes au développement d'une évaluation réellement au service des apprentissages, sans doute seraient-ils plus enclins à participer à des recherches collaboratives dont les apports en termes de développement professionnel semblent être très intéressants (Morissette et Tessaro, 2016).

\section{Références bibliographiques}

Allal, L. et Mottier Lopez, L. (2005). L'évaluation formative de l'apprentissage : revue de publications en langue française. Dans Centre pour la recherche et l'innovation dans l'enseignement (dir.), L'évaluation formative pour un meilleur apprentissage dans les classes secondaires (p. 265-290). Paris : Edition OCDE.

Blais, M. et Martineau, S. (2006). L'analyse inductive générale : Description d'une démarche visant à donner un sens à des données brutes. Recherches Qualitatives, 26(2), 1-18.

Bourgeois, L. et Laveault, D. (2015). Évaluation par les pairs à l'écrit: qualité des rétroactions pour soutenir la phase de révision. Dans P.-F. Coen et L. M. Bélair (dir.), Évaluation et autoévaluation. Quels espaces de formation? (p. 101-117). Louvain-laNeuve : De Boeck Supérieur.

Butori, R. et Parguel, B. (2010). Les biais de réponse. Impact du mode de collecte des données et de l'attractivité de l'enquêteur. AFM, France. Disponible en ligne sur: https://halshs.archives-ouvertes.fr/halshs-00636228/document.

Campanale, F. (2007). Évaluation réflexive en formation professionnelle et évaluation interactive dans les classes. Dans L. Allal et L. Mottier Lopez. (dir.), Régulation des apprentissages en situation scolaire et en formation (p. 191-206). Bruxelles : De Boeck.

Conseil National d'Évaluation du Système Scolaire (Cnesco) (2014). L'évaluation des élèves par les enseignants dans la classe et les établissements : réglementation et pratiques. Une comparaison internationale dans les pays de l'OCDE. Paris : Cnesco.

Crahay, M. (dir.) (2013). L'école peut-elle être juste et efficace? De l'égalité des chances à l'égalité des acquis. Bruxelles : De Boeck.

Crahay, M., Wanlin, P., Issaieva, E. et Laduron, I. (2010). Fonctions, structuration et évolution des croyances (et connaissances) des enseignants. Une revue critique de la littérature anglo-saxonne. Note de synthèse. Revue française de pédagogie, 172, 85-129.

Décret «Missions » : décret de la Communauté française du 24 juillet 1997 définissant les missions prioritaires de l'enseignement fondamental et de l'enseignement secondaire et organisant les structures propres à les atteindre (1997). Moniteur belge, 23 septembre, p. 24653.

De Stercke, J., Renson, J.-M., Cambier, J.-B., Leemans, M., Maréchal, C., Radermaecker, G., Temperman, G., Beckers, J. et De Lièvre, B. (2010). Dynamiser l'insertion professionnelle des enseignants débutants du secondaire. Disponible en ligne sur: http://www.enseignement.be/index.php?page $=23827$ etdo_id $=8042$ etdo_check $=$.

Detroz, P., Crahay, M. et Fagnant, A. (dir.) (2017). L'évaluation à la lumière des contextes et des disciplines. Bruxelles : De Boeck. 
Devos, C. et Paquay, L. (2013). Le choc de la pratique chez les enseignants débutants : Quelle place pour la réflexivité ? Dans M. Altet, J. Desjardins, R. Etienne, L. Paquay et P. Perrenoud (dir.), Former des enseignants réflexifs. Obstacles et résistances (p. 229-247). Bruxelles : De Boeck.

Durand, M. (1996). L'enseignement en milieu scolaire. Paris : PUF.

Fagnant, A., Etienne, R., Mottier Lopez, L. et Hindryckx, M-N. (soumis). L'évaluation comme objet d'apprentissage et comme outil de développement professionnel dans le cadre de la formation d'enseignants. Évaluer. Journal International de Recherche en Éducation et Formation.

Fagnant, A. et Goffin, C. (à paraître). Les conceptions des futurs enseignants du secondaire en matière d'évaluation : entre un accord de principe et une vision limitée de l'évaluation formative. Mesure et Évaluation en Éducation, 40(1).

Huberman, M. (1989). Les phases de la carrière enseignante. Un essai de description et de prévision. Revue française de Pédagogie, 86, 5-16.

Issaieva, E. et Crahay, M. (2010). Conception de l'évaluation scolaire des élèves et des enseignants : validation d'échelles et études de leurs relations. Mesure et Évaluation en Éducation, 33(1), 31-62.

Issaieva E., Yerly, G., Petkova, I., Marbaise, C. et Crahay, M. (2015). Conceptions et prises de positions des enseignants face à l'évaluation scolaire dans quatre systèmes éducatifs européens : quel est le reflet des cultures et politiques éducatives ? Dans P.-F. Coen et L. M. Bélair (dir.), Évaluation et autoévaluation. Quels espaces de formation? (p. 73-98). Louvain-la-Neuve : De Boeck Supérieur.

Luisoni, M. et Monnard, I. (2015). L'évolution des conceptions de l'évaluation scolaire chez les futurs enseignants en formation initiale. Dans P.-F. Coen et L. M. Bélair (dir.), Évaluation et autoévaluation. Quels espaces de formation? (p. 175-196). Louvain-laNeuve : De Boeck Supérieur.

Morissette, J. (2010). Un panorama de la recherche sur l'évaluation formative des apprentissages. Mesure et Évaluation en Éducation, 33(3), 1-27.

Morissette, J. et Tessaro, W. (2016) Apports des recherches collaboratives sur le développement professionnel des enseignants et des formateurs en matière d'évaluation. Evaluer. Journal International de Recherche en Éducation et Formation, 1(2), 9-14.

Mottier Lopez, L. (2015). Evaluations formative et certificative des apprentissages. Louvainla-Neuve : De Boeck.

Mottier Lopez, L. et Laveault, D. (2008). L'évaluation des apprentissages en contexte scolaire : développements, enjeux et controverses. Mesure et Évaluation en Éducation, $31(3), 5-34$.

Ocdé (2005). L'évaluation formative. Pour un meilleur apprentissage dans les classes secondaires. Paris : Édition OCDE.

Ocdé (2013). Résultats de TALIS 2013. Une perspective internationale sur l'enseignement et l'apprentissage. Paris : Édition OCDE.

Parmentier, P. et Paquay, L. (2002). En quoi les situations d'enseignement/apprentissage favorisent-elles le développement de compétences? Vers un outil d'analyse : le CompAS. Louvain-la-Neuve.

Paquay, L. (1994). Vers un référentiel des compétences professionnelles de l'enseignant? Recherche et Formation, 16, 7-38.

Rey, O. et Feyfant, A. (2014). Evaluer pour (mieux) faire apprendre. Dossier de veille de l'Institut Français de l'Éducation, 94, 1-44.

Soussi, A., Ducrey, F., Ferrez, E., Nidegger, C. et Viry, G. (2006). Pratiques d'évaluation : ce qu'en disent les enseignants (à l'école obligatoire et dans l'enseignement postobligatoire général). Rapport du service de la recherche en éducation, Université de Genève. 
Disponilbe en ligne sur le serveur suisse de documents pour l'éducation et la formation : http://edudoc.ch/record/3603/.

Vanhulle, S. (2002). Des savoirs en jeu au savoir en " je ». Médiations sociales et processus de subjectivation en formation initiale d'enseignants. Cahiers du Service de Pédagogie expérimentale, 11-12, 49-62.

Vantourout, M. et Goasdoué, R. (2014). Approches et validité psycho-didactique des évaluations. Éducation et Formation, e-302, 139-156.

Vause, A. (2010). Les croyances et connaissances des enseignants de l'école primaire à propos de l'acte d'enseigner. Éducation et Formation, 294, 14-19.

Younès, N. et Gaime, E. (2012). L'évaluation formative en contexte : points de vue d'enseignants, points de vue d'élèves. Diversité, VEI, 161-166. 